\title{
Impact of temporal resolution on estimating capillary RBC-flux with optical coherence tomography
}

\author{
Baoqiang Li \\ Hui Wang \\ Buyin $\mathrm{Fu}$ \\ Ruopeng Wang \\ Sava Sakadžić \\ David A. Boas
}




\title{
Impact of temporal resolution on estimating capillary RBC-flux with optical coherence tomography
}

\author{
Baoqiang Li, ${ }^{a, *}$ Hui Wang, ${ }^{a, b}$ Buyin Fu, ${ }^{a}$ Ruopeng Wang, ${ }^{b}$ Sava Sakadžić, ${ }^{a}$ and David A. Boas ${ }^{a}$ \\ ${ }^{a}$ Massachusetts General Hospital/Harvard Medical School, Optics Division, Athinoula A. Martinos Center for Biomedical Imaging, Charlestown, \\ Massachusetts 02129, United States \\ ${ }^{b}$ Massachusetts General Hospital/Harvard Medical School, Laboratory for Computational Neuroimaging, Athinoula A. Martinos Center for \\ Biomedical Imaging, Charlestown, Massachusetts 02129, United States
}

\begin{abstract}
Optical coherence tomography (OCT) has been used to measure capillary red blood cell (RBC) flux. However, one important technical issue is that the accuracy of this method is subject to the temporal resolution $(\Delta t)$ of the repeated RBC-passage B-scans. A ceiling effect arises due to an insufficient $\Delta t$ limiting the maximum RBC-flux that can be measured. In this letter, we first present simulations demonstrating that $\Delta t=1.5 \mathrm{~ms}$ permits measuring RBC-flux up to $150 \mathrm{RBCs} / \mathrm{s}$ with an underestimation of $9 \%$. The simulations further show that measurements with $\Delta t=3$ and $4.5 \mathrm{~ms}$ provide relatively less accurate estimates for typical physiological fluxes. We provide experimental data confirming the simulation results showing that reduced temporal resolution (i.e., a longer $\Delta t$ ) results in an underestimation of mean flux and compresses the distribution of measured fluxes, which potentially confounds physiological interpretation of the results. The results also apply to RBC-passage measurements made with confocal and two-photon microscopy for estimating capillary RBC-flux. ๑ 2017 Society of Photo-Optical Instrumentation Engineers (SPIE) [DOI: 10.1117/1.JBO.22.1.016014]
\end{abstract}

Keywords: capillary blood flow; RBC-flux; optical coherence tomography; temporal resolution.

Paper 160745LR received Oct. 29, 2016; accepted for publication Jan. 9, 2017; published online Jan. 26, 2017.

\section{Introduction}

Understanding cerebral microvascular flow regulation and capacity to deliver oxygen to cortical tissue is of critical importance in assessing brain physiology and pathophysiology. ${ }^{1}$ In addition, cerebral cortical microvascular networks play a vital role in delivering oxygen and nutrients to support brain cognitive functions. ${ }^{2}$ Recent studies suggest that heterogeneity in capillary flow either passively correlates with ${ }^{3}$ or actively regulates ${ }^{4}$ oxygen extraction. ${ }^{2,5}$ To further understand the properties of the cerebral microcirculation, optical techniques,- - for example, two-photon microscopy (TPM) and more recently, optical coherence tomography (OCT) — have been advanced to investigate the highly heterogeneous and dynamic capillary properties at high spatiotemporal resolution, and preferably over many capillaries simultaneously for strengthening the statistical power of the analysis. ${ }^{6,7}$

The OCT-based RBC-passage technique detects reflected optical intensity changes due to RBCs passing through the optical focal volume. ${ }^{7}$ Because of the high spatial sampling rate of OCT, this technique has the ability of measuring absolute RBC-flux of multiple capillaries simultaneously, ${ }^{7,8}$ which makes it suitable for characterizing the distribution of capillary flux in microvascular networks and neurocapillary coupling. ${ }^{9,10}$ However, an insufficient temporal resolution $(\Delta t)$ of this RBCpassage technique may fail in capturing RBCs with very high speed when passing the focal volume, inducing aliasing in the measurements, consequently confounding physiological interpretation of the results. The aim of this study is to investigate how $\Delta t$ impacts the accuracy in estimating the capillary flux and

*Address all correspondence to: Baoqiang Li, E-mail: baoqiang.li@mgh. harvard.edu to provide general guidance for the $\Delta t$ requirement when measuring the distribution of flux and/or mean flux of cortical capillaries in rodents by the OCT RBC-passage technique. Our simulations show that $\Delta t=1.5 \mathrm{~ms}$ underestimates the flux with an underestimation of $\sim 9 \%$, at the true flux (synthetized with $\Delta t=0.75 \mathrm{~ms}$ ) of $150 \mathrm{RBCs} / \mathrm{s}$; in contrast, $\Delta t=3$ and $4.5 \mathrm{~ms}$ cause an underestimation of $\sim 57 \%$ and $\sim 74 \%$, respectively. At the true flux of $130 \mathrm{RBCs} / \mathrm{s}, \Delta t=1.5,3$, and 4.5 cause an underestimation of $\sim 6 \%, \sim 50 \%$, and $\sim 70 \%$, respectively. Furthermore, experimental data with either $\Delta t=$ 0.75 or $1.5 \mathrm{~ms}$ were acquired in two separate groups of mice for evaluating the sufficiency of $\Delta t=1.5 \mathrm{~ms}$. The experimental results show that with $\Delta t=1.5 \mathrm{~ms}$ the measured flux was underestimated by $\sim 5 \%$ at $\sim 130 \mathrm{RBCs} / \mathrm{s}$ compared with the flux measured with $\Delta t=0.75 \mathrm{~ms}$, which was the maximum flux that was measured in our experiments. This observed $5 \%$ underestimation of flux at $130 \mathrm{RBCs} / \mathrm{s}$ is very close to our simulation result. The downsampled experimental fluxes with $\Delta t=$ 3 and $4.5 \mathrm{~ms}$ showed the compressed distribution of flux and the reduction in mean flux, which we also observed in the simulations. Other studies reported that a resting capillary flux of $>150 \mathrm{RBCs} / \mathrm{s}$ in rodents was very rare, ${ }^{11-14}$ therefore, $\Delta t=1.5 \mathrm{~ms}$ is expected to permit accurately characterizing the distribution of flux and/or the mean flux in cortical capillaries.

\section{Materials and Methods}

\subsection{Animals}

All animal experimental procedures were reviewed and approved by the Massachusetts General Hospital Subcommittee

$1083-3668 / 2017 / \$ 25.00$ @ 2017 SPIE 
on Research Animal Care. We used CD1-Elite mice (Charles River Laboratories, $n=10$, male, 3 to 4 months old, $\sim 35 \mathrm{~g}$ ) in this study. All surgical procedures, including the sealed cranial window preparation and the cannulation of femoral artery, were conducted under $1.5 \%$ to $2 \%$ isoflurane anesthesia in air $/ \mathrm{O}_{2}$ mixture. In experiments, mice $(n=10)$ were separated into two groups: group $1\left(n_{1}=6\right)$ and group $2\left(n_{2}=4\right)$. Acquisition was conducted under $\alpha$-chloralose administered intravenously. ${ }^{10}$ Body temperature was maintained at $\sim 37^{\circ} \mathrm{C}$ throughout all experiments. In experiments, the mean systemic arterial blood pressure and arterial partial pressure of $\mathrm{O}_{2}$ and $\mathrm{CO}_{2}$ averaged over $n=10$ mice were $\sim 93,96$, and $35 \mathrm{mmHg}$, respectively; the $\mathrm{pH}$ of systemic arterial blood was $\sim 7.36$.

\subsection{Spectral-Domain Optical Coherence Tomography}

Capillary RBC-passage B-scans were conducted using an optimized commercial spectral-domain OCT (SD-OCT) system (Thorlabs Inc.). ${ }^{7}$ The light source has a central wavelength of $1310 \mathrm{~nm}$ with a 170-nm bandwidth yielding an axial resolution of $3.5 \mu \mathrm{m}$ in brain tissue. The transverse resolution was $3.5 \mu \mathrm{m}$ when using the $10 \times$ objective (NA $=0.26$ ). This SD-OCT system has a maximum acquisition rate of 47,000 A-lines/s.

\subsection{Determination of the Red Blood Cell Flux}

The details of the RBC-passage technique have been described elsewhere. ${ }^{7}$ Figures 1(a) and 1(b) help explain the acquisition protocol of the RBC-passage B-scan. Figure 1(a) displays a picture of a typical cranial window over mouse cortex. As shown by the red lines in Fig. 1(a), three lines were typically selected in the cranial window in each mouse for conducting the OCT RBC-passage B-scans. Each RBC-passage B-scan consisted of 30 or 60 A-lines and spanned a distance of 50 or $100 \mu \mathrm{m}$ in the $X-Z$ cross-sectional plane, which yields $\Delta t=\sim 0.75$ or $\sim 1.5 \mathrm{~ms}$, respectively. Each line was consecutively scanned for 2 min. Because the OCT speckle fluctuation in rodent cortex is mainly due to the motion of RBCs, ${ }^{15}$ capillaries [bright pixels in Fig. 1(b)] could be identified and manually selected based on the B-scan variance images. For each selected capillary, the pixel intensities at the same coordinate of the consecutive B-scans were extracted to reveal the RBC-passage time course. Here, we used a custom-developed MATLAB graphic user interface to make sure the selected points were in capillaries by selecting points that had the largest intensity fluctuations. Next, the RBCpassage time courses were denoised by a temporal 1-D Gaussian filter with standard deviation $\sigma=1.5 \mathrm{~ms}$ to reduce false-positive noise peaks when counting the RBC passages. The $\sigma$ relates to the FWHM as FWHM $=2 \sqrt{2 \ln 2} \sigma$, so, $\sigma=1.5 \mathrm{~ms}$ would yield a FWHM of $\sim 3.5 \mathrm{~ms}$. The RBC-flux of $200 \mathrm{RBCs} / \mathrm{s}$ corresponds to an RBC passing every $5 \mathrm{~ms}$ in average. Thus, using a Gaussian filter with FWHM $=3.5 \mathrm{~ms}$, we expect to diminish the noise but not mask the RBC passages when RBCs pass every $5 \mathrm{~ms}$. Such a high flux of $200 \mathrm{RBCs} / \mathrm{s}$ was observed, which was driven by high RBC linear density instead of high speed. ${ }^{14}$ The RBC-passage time courses were then normalized with the range from 0 to 1 (i.e., the minimum value was subtracted from the time course then the time course was normalized by the maximum value). A MATLAB function "findpeaks" was used to count the RBC-passage peaks by employing a threshold set at the half-maximum intensity (i.e., the threshold was 0.5 after the time courses were normalized by the maximum value) of each RBC-passage time course. The number of RBC-passage peaks was averaged over the 2-min scan for each capillary to obtain a mean flux.

The performance of the RBC-passage peak counting procedure was evaluated by simulations. Briefly, speckle noise (filtered from experimental data) was added to synthetized RBC passages (described in Sec. 2.4). Peak counting was performed on those RBC passages with and without filtering $(\sigma=1.5 \mathrm{~ms})$. Without filtering, the ratio of the estimated flux to true flux was $\sim 179 \%$ due to false detections associated with noise. With filtering, the ratio decreased to $\sim 101 \%$.

\subsection{Numerical Modeling of Red Blood Cell Flux}

We modeled the intensity change due to RBC passage in a virtual capillary by a Gaussian function: $f\left(t^{\prime}\right)=\exp \left[-\frac{\left(t^{\prime}-t_{o}\right)^{2}}{2 \sigma^{2}}\right]$,

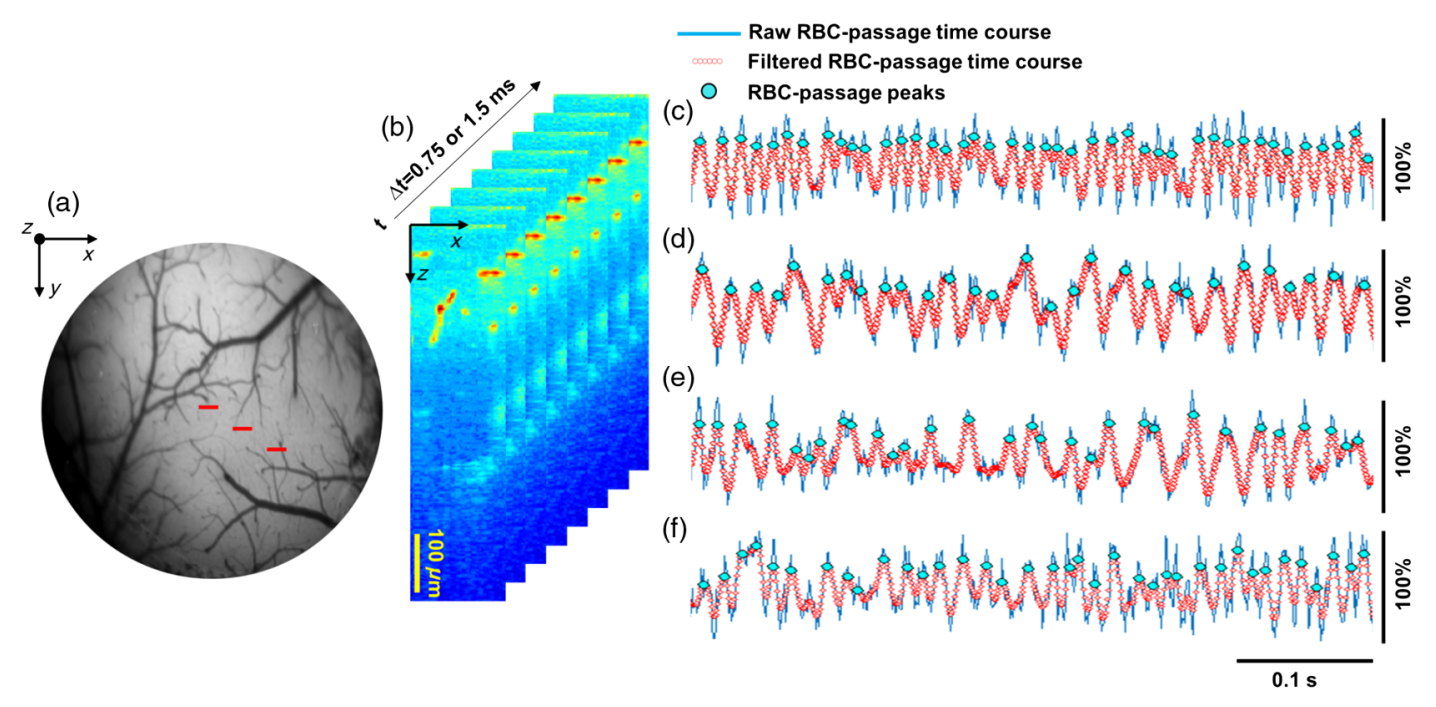

Fig. 1 (a) The picture displays a typical cranial window showing the vasculature of the cortical surface. The red lines indicate where repeated B-scans were performed to obtain the RBC-passage time courses. (b) Interlaced B-scan images illustrate the repetitive RBC-passage B-scan. (c)-(f) Representative RBCpassage time courses $(0.5 \mathrm{~s})$ of four different capillaries are presented. 
where $t^{\prime}$ represents a time point in an RBC-passage time course; $t_{0}$ is the center time point of a single RBC passage. According to the previous study, ${ }^{7}$ the $\mathrm{RBC}$-speed $\left(\mathrm{V}_{\mathrm{RBC}}\right)$ relates to the $\mathrm{RBC}$ diameter $\left(\phi_{\mathrm{RBC}}\right)$ and the Gaussian FWHM by $V_{\mathrm{RBC}}=\frac{\phi_{\mathrm{RBC}}}{2 \sqrt{2 \ln 2} \sigma}$. Here, we set $\phi_{\mathrm{RBC}}$ in our simulations to $6.5 \mu \mathrm{m}$. $^{7}$ As reported, ${ }^{14}$ the flux $\left(f_{\mathrm{RBC}}\right)$ relates to $V_{\mathrm{RBC}}$ and the distance between neighboring $\mathrm{RBCs}\left(d_{\mathrm{RBC}}\right)$ as $f_{\mathrm{RBC}}=V_{\mathrm{RBC}} / d_{\mathrm{RBC}}$. Then we numerically investigated the RBC-passage time courses for a range of $V_{\mathrm{RBC}}$ and $d_{\mathrm{RBC}}$. Specifically, $40 V_{\mathrm{RBC}}$ were evenly selected between 0.1 and $3.5 \mathrm{~mm} / \mathrm{s}$, which covers the range of capillary $V_{\mathrm{RBC}}$ reported in the literature. ${ }^{11-14,16-26}$ The mean value of $\left\langle d_{\mathrm{RBC}}\right\rangle$ was reported to be $14 \pm 2 \mu \mathrm{m} .{ }^{14}$ In simulation, 20 different $\left\langle d_{\mathrm{RBC}}\right\rangle$ were evenly chosen between 12 and $26 \mu \mathrm{m}$. For each RBC-passage time course, $V_{\mathrm{RBC}}$ is fixed for all the RBCs in that time course; and the specific $d_{\mathrm{RBC}}$ between neighboring RBCs was uniformly distributed around a certain mean $\left\langle d_{\mathrm{RBC}}\right\rangle$. So, combinations of $40 V_{\mathrm{RBC}}$ and $20\left\langle d_{\mathrm{RBC}}\right\rangle$ result in $40 \times 20=800$ RBC-passage time courses. Every RBC-passage time course was generated for 2 min of simulation time with $\Delta t=0.75 \mathrm{~ms}$. Each of the $800 \mathrm{RBC}$-passage time courses was regenerated 100 times with different random $\mathrm{RBC}$ distributions for averaging. In order to investigate the impact of $\Delta t$, each of the $800 \times 100=80,000$ RBC-passage time courses with $\Delta t=0.75 \mathrm{~ms}$ was downsampled to $\Delta t=1.5,3$, and $4.5 \mathrm{~ms}$. With these four different $\Delta t$ 's, a total of $4 \times 80,000=$ 320,000 RBC-passage time courses were obtained; each of them yielded a mean flux $\left\langle f_{\mathrm{RBC}}\right\rangle$. The $\left\langle f_{\mathrm{RBC}}\right\rangle$ from the individual RBC-passage time courses was binned for comparing results with different $\Delta t$ 's.

\section{Results}

Representative RBC-passage time courses (0.5-s trace) of four different capillaries are shown in Figs. 1(c)-1(f). For the four representative capillaries, the mean fluxes averaged over the 2-min scan are 91, 60, 56, and $75 \mathrm{BBCs} / \mathrm{s}$, respectively.

\subsection{Effect of B-Scan Temporal Resolution: Numerical Modeling}

We first investigate the impact of $\Delta t$ on the estimation of flux by numerical modeling. Figures 2(a)-2(c) show three synthetized RBC-passage time courses (0.2-s trace). In these three time courses, the mean $d_{\mathrm{RBC}}$ was fixed at $14 \mu \mathrm{m}$; but the individual $d_{\mathrm{RBC}}$ between neighboring RBCs were randomly varied around $14 \mu \mathrm{m}$. In Figs. 2(a)-2(c), the $V_{\mathrm{RBC}}$ is 2,1 , and $0.8 \mathrm{~mm} / \mathrm{s}$, and the resultant flux is 100,50 , and $40 \mathrm{RBCs} / \mathrm{s}$, respectively. The RBC-passage time courses with $\Delta t=0.75 \mathrm{~ms}$ are in red; and the $\Delta t=4.5 \mathrm{~ms}$ in blue. This illustration indicates that $\Delta t=4.5 \mathrm{~ms}$ might be appropriate for accurate estimation of flux when the $V_{\mathrm{RBC}}<0.8 \mathrm{~mm} / \mathrm{s}$ but underestimates flux when $V_{\mathrm{RBC}}>1 \mathrm{~mm} / \mathrm{s}$.

To quantify this ceiling effect with simulations, we synthetized RBC-passage time courses with different combinations of $V_{\mathrm{RBC}}$ and $d_{\mathrm{RBC}}$ (see details in Sec. 2.4). The comparison between the simulated fluxes with $\Delta t=0.75 \mathrm{~ms}$ and the fluxes with $\Delta t=0.75,1.5,3$, and $4.5 \mathrm{~ms}$ is presented in Fig. 2(d). Here, the $x$-axis is the simulated flux with $\Delta t=0.75 \mathrm{~ms}$; the $y$-axis is the simulated fluxes with $\Delta t=0.75,1.5,3$, and $4.5 \mathrm{~ms}$. The green line shows flux with $\Delta t=0.75 \mathrm{~ms}$, which serves as a reference for comparison. Ideally, the estimated flux should match the true flux. We expect that as $\Delta t$ becomes longer that the estimated flux will underestimate the true value, particularly at large flux values. Comparing with the fluxes with $\Delta t=0.75 \mathrm{~ms}$, Fig. 2(d) shows that with $\Delta t=1.5,3$, and $4.5 \mathrm{~ms}$, the fluxes are underestimated by $9 \%, 57 \%$, and $74 \%$, respectively, at the true flux of $150 \mathrm{RBCs} / \mathrm{s}$. At the true flux of $130 \mathrm{RBCs} / \mathrm{s}, \Delta t=1.5,3$, and 4.5 cause an underestimation of $\sim 6 \%, \sim 50 \%$, and $\sim 70 \%$, respectively.

\subsection{Effect of B-Scan Temporal Resolution: Experimental Data}

In experiments, the RBC-passage B-scans were performed in mice of group $1\left(n_{1}=6\right)$ with $\Delta t=1.5 \mathrm{~ms}$, and group 2 $\left(n_{2}=4\right)$ with $\Delta t=0.75 \mathrm{~ms}$. In both groups, the measurements were obtained at cortical depths up to $\sim 700 \mu \mathrm{m}$ but mainly around $\sim 500 \mu \mathrm{m}$. The original RBC-passage time courses of group 1 that were acquired with $\Delta t=1.5 \mathrm{~ms}$ were downsampled to get two additional datasets with $\Delta t=3$ and $4.5 \mathrm{~ms}$. Similarly, the original RBC-passage time courses of group 2 that were acquired with $\Delta t=0.75 \mathrm{~ms}$ were downsampled to get three additional datasets with $\Delta t=1.5,3$, and $4.5 \mathrm{~ms}$. We then calculated the mean flux for each RBC-passage time course with different $\Delta t$ 's to investigate the impact of $\Delta t$. Figure 2(e) shows
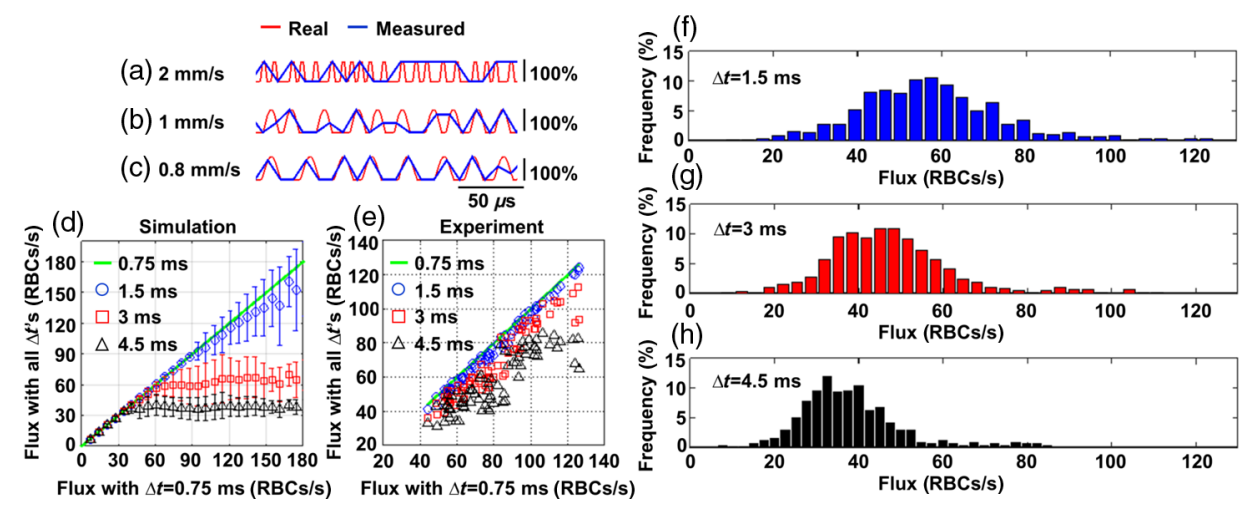

Fig. 2 (a)-(c) Illustration of aliasing with simulated RBC-passage time courses with $V_{\mathrm{RBC}}=2,1$ and $0.8 \mathrm{~mm} / \mathrm{s}$, respectively. The "true" time courses $(\Delta t=0.75 \mathrm{~ms})$ are in red, while the downsampled ones $(\Delta t=4.5 \mathrm{~ms})$ are in blue. (d) Comparison between simulated fluxes with $\Delta t=0.75 \mathrm{~ms}$, and the simulated fluxes with all $\Delta t$ 's $(0.75,1.5,3$, and $4.5 \mathrm{~ms})$. (e) Comparison between experimental fluxes with $\Delta t=0.75 \mathrm{~ms}$, and the experimental fluxes with all $\Delta t$ 's $(0.75,1.5,3$, and $4.5 \mathrm{~ms})$. (f)-(h) Histograms of experimental fluxes with $\Delta t=1.5,3$, and $4.5 \mathrm{~ms}$. 
fluxes measured in group 2. In Fig. 2(e), the $X$-axis presents the experimental fluxes with $\Delta t=0.75 \mathrm{~ms}$, which served as the "truth" in the experimental scenario; and the $Y$-axis is the fluxes with $\Delta t=0.75,1.5,3$, and $4.5 \mathrm{~ms}$. Comparing with the fluxes with $\Delta t=0.75 \mathrm{~ms}$, Fig. 2(e) showed that fluxes with $\Delta t=$ $1.5 \mathrm{~ms}$ are accurately estimated with an underestimation of $\sim 5 \%$ at $130 \mathrm{RBCs} / \mathrm{s}$, which is the maximum flux measured in our experiments. In contrast, with $\Delta t=3$ and $4.5 \mathrm{~ms}$, the fluxes are underestimated by $19 \%$ and $42 \%$, respectively, at the flux of $130 \mathrm{RBCs} / \mathrm{s}$.

The histograms in Figs. 2(f)-2(h) present the distribution of flux measured in 313 capillaries over $n=10$ mice. As shown, when $\Delta t$ becomes longer, the distribution of flux becomes more compressed and left-shifted to lower fluxes, as would be expected due to aliasing introducing a ceiling effect. The mean flux is 57,47 , and $38 \mathrm{RBCs} / \mathrm{s}$, with $\Delta t=1.5,3$, and $4.5 \mathrm{~ms}$, respectively, showing a reduction of the mean flux with longer $\Delta t$ 's.

\section{Discussion and Conclusion}

We investigated the impact of temporal resolution on the accuracy of estimating RBC-flux based on the OCT RBC-passage technique. Simulations show that with $\Delta t=1.5 \mathrm{~ms}$, the flux is underestimated by $9 \%$ for fluxes up to $150 \mathrm{RBCs} / \mathrm{s}$ and becomes worse for larger fluxes. For longer $\Delta t$ 's, aliasing contaminates the measurements and introduces a significant underestimation of flux. Specifically, with $\Delta t=3.0$ and $4.5 \mathrm{~ms}$, the flux is underestimated by $57 \%$ and $74 \%$, respectively, at the true flux of $150 \mathrm{RBCs} / \mathrm{s}$.

Capillary fluxes were experimentally measured in two groups of mice (total $n=10$ ) with $\Delta t=1.5$ and $0.75 \mathrm{~ms}$ in groups 1 and 2 , respectively. The results demonstrate that with $\Delta t=1.5,3$, and $4.5 \mathrm{~ms}$, the flux is underestimated by $5 \%, 19 \%$, and $42 \%$, respectively, at the maximum flux of $130 \mathrm{RBCs} / \mathrm{s}$ that was measured with $\Delta t=0.75 \mathrm{~ms}$. Simulation shows a similar trend in that $\Delta t=1.5,3$, and 4.5 cause an underestimation of $\sim 6 \%, \sim 50 \%$, and $\sim 70 \%$, at the true flux of $130 \mathrm{RBCs} / \mathrm{s}$, respectively. The similarity of the simulation and experimental results support our interpretation that the underestimation of flux with longer $\Delta t$ 's arises from aliasing. This aliasing results in misinterpretation of the RBC-flux characteristics, as illustrated in the histograms presented in Figs. 2(f)-2(h), revealing a compression of the distribution of flux as well as a reduction in the mean flux with longer $\Delta t$ 's.

One may argue that shorter $\Delta t$ than $1.5 \mathrm{~ms}$, e.g., $\Delta t=0.75 \mathrm{~ms}$, will enable accurately measuring higher fluxes. However, shorter $\Delta t$ means smaller FOV, in turn, fewer measurements. On the contrary, longer $\Delta t$ will allow a larger FOV and more measurements, which benefits a more powerful statistical analysis. Therefore, not readily pushing the speed limit of acquisition, we suggested that $\Delta t=1.5 \mathrm{~ms}$ could be necessarily fast to measure typical capillary flux in mice cortex.

Some limitations exist in this study. First, while we found qualitative agreement between the simulations and experiments, some discrepancies can be observed between Figs. 2(d) and 2(e). The simulation in Fig. 2(d) showed a more pronounced and earlier ceiling effect with longer $\Delta t$ 's. This could be a result of the simulation parameters, e.g., the $V_{\mathrm{RBC}}$ and $d_{\mathrm{RBC}}$, being different from the experimental values. For example, $d_{\mathrm{RBC}}$ might be distributed differently than the range used in our simulations. Experimentally, there could be capillaries with low $V_{\mathrm{RBC}}$ and small $d_{\mathrm{RBC}}$, thus resulting in a high flux that is still resolvable despite a long $\Delta t$. As an example, capillary flux as high as $\sim 200 \mathrm{RBCs} / \mathrm{s}^{14}$ was reported; but such a high flux was driven by a high RBC linear density instead of high speed $(\sim 1.5 \mathrm{~mm} / \mathrm{s})$, which would be detectable with $\Delta t=1.5 \mathrm{~ms}$. Indeed, many studies have reported that the RBC-speed within most cortical capillaries is $<2 \mathrm{~mm} / \mathrm{s},{ }^{11-14,16-26}$ and thus, passage of a $6.5-\mu \mathrm{m}$ RBC would be $>3 \mathrm{~ms}$, indicating that associated fluxes can be measured with $\Delta t=1.5 \mathrm{~ms}$. Another possibility for this discrepancy is due to the statistics of the measurement noise in the experimental data. Practically, the noise level increases with depth because of optical attenuation. As a result, the filter parameter should be adjusted to adapt to the varying signal-to-noise ratio. Although a constant filter parameter $(\sigma=1.5 \mathrm{~ms})$ has been used to avoid bias in analysis, it might result in a depth-dependent noise reduction. Therefore, the flux would likely be measured more accurately in some superficial capillaries while overestimated in deeper capillaries because of false positives resulting from stronger noise. Second, with the experimental fluxes, we simply evaluated the underestimation of the estimated flux at a downsampled $\Delta t$ relative to the flux with the fastest $\Delta t=0.75 \mathrm{~ms}$, as we did not know the true flux for the experimental data.

Another limitation of our RBC-passage technique is that we could not distinguish whether or not a dual-peak RBC-passage was because of random orientation of a flowing $\mathrm{RBC}$ or two RBCs flowing together. Such a dual-peak RBC-passage might be counted as one in estimating the flux because of the potential undersampling induced by fast RBCs; further, given the filter parameter used $(\sigma=1.5 \mathrm{~ms})$, RBCs need to be spaced by more than $1.5 \mathrm{~ms}$ to be resolved as separate RBCs.

Last, we acknowledge that some capillaries with very high speed, e.g., thoroughfare capillaries, can exist ${ }^{18,27}$ and that the associated fluxes may not be accurately measured with $\Delta t=1.5 \mathrm{~ms}$. Therefore, $\Delta t=1.5 \mathrm{~ms}$ would be more appropriate for estimating the mean flux of the majority of capillaries with many branch orders between arterioles and venules, but possibly not sufficient for the relatively rare thoroughfare capillaries. ${ }^{16}$ Note that possible thoroughfare capillaries are rare and are often located near the brain surface (e.g., 50 to $70 \mu \mathrm{m}) ;^{16,18,27}$ we thus expect that those rare cases do not have a large impact in characterizing the distribution of flux and the mean flux when measuring a large ensemble of capillaries.

In summary, we investigated the impact of temporal resolution on the accuracy of estimating capillary RBC-flux. Simulations and experiments characterized this impact. Our findings support that $\Delta t=1.5 \mathrm{~ms}$ permits accurate measurements of RBC-flux up to $150 \mathrm{RBCs} / \mathrm{s}$. Longer $\Delta t$ 's will result in a ceiling effect that causes underestimation of physiologically reasonable flux values. These results should serve as a general rule in determining the temporal resolution that is necessary to measure the distribution of flux and the mean flux in cortical capillaries in mice. These results are not limited to OCT but also extend to other optical scanning techniques for estimating RBC passage, including confocal microscopy and TPM.

\section{Disclosures}

The authors declare no conflicts of interest.

\section{Acknowledgments}

This study was supported by the National Institutes of Health grants (R01-EB021018, P01-NS055104, and R01-NS091230). 
This study was also supported by the Air Force Office of Sponsored Research (AFOSR FA-9550-15-1-0473).

\section{References}

1. C. Iadecola, "Neurovascular regulation in the normal brain and in Alzheimer's disease," Nat. Rev. Neurosci. 5, 347-360 (2004).

2. S. N. Jespersen and L. Østergaard, "The roles of cerebral blood flow, capillary transit time heterogeneity, and oxygen tension in brain oxygenation and metabolism," J. Cereb. Blood Flow Metab. 32, 264-277 (2012)

3. T. J. Huppert et al., "A multicompartment vascular model for inferring baseline and functional changes in cerebral oxygen metabolism and arterial dilation," J. Cereb. Blood Flow Metab. Off. J. Int. Soc. Cereb. Blood Flow Metab. 27, 1262-1279 (2007).

4. C. N. Hall et al., "Capillary pericytes regulate cerebral blood flow in health and disease," Nature 508, 55-60 (2014).

5. P. M. Rasmussen, S. N. Jespersen, and L. Østergaard, "The effects of transit time heterogeneity on brain oxygenation during rest and functional activation," J. Cereb. Blood Flow Metab. 35, 432-442 (2015).

6. W. S. Kamoun et al., "Simultaneous measurement of RBC velocity, flux, hematocrit and shear rate in vascular networks," Nat. Methods 7, 655-660 (2010).

7. J. Lee et al., "Multiple-capillary measurement of RBC speed, flux, and density with optical coherence tomography," J. Cereb. Blood Flow Metab. 33, 1707-1710 (2013).

8. H. Ren et al., "Quantitative imaging of red blood cell velocity in vivo using optical coherence Doppler tomography," Appl. Phys. Lett. 100, 233702 (2012).

9. J. Lee, W. Wu, and D. A. Boas, "Early capillary flux homogenization in response to neural activation," J. Cereb. Blood Flow Metab. 36, 375-380 (2015)

10. B. Li et al., "Contribution of low- and high-flux capillaries to slow hemodynamic fluctuations in the cerebral cortex of mice," J. Cereb. Blood Flow Metab. 36, 1351-1356 (2016).

11. A. Villringer et al., "Capillary perfusion of the rat brain cortex. An in vivo confocal microscopy study," Circ. Res. 75, 55-62 (1994).

12. D. Kleinfeld, "Cortical blood flow through individual capillaries in rat vibrissa S1 cortex: stimulus-induced changes in flow are comparable to the underlying fluctuations in flow," Int. Congr. Ser. 1235, 115-122 (2002).

13. I. Krolo and A. G. Hudetz, "Hypoxemia alters erythrocyte perfusion pattern in the cerebral capillary network," Microvasc. Res. 59, 72-79 (2000).
14. D. Kleinfeld et al., "Fluctuations and stimulus-induced changes in blood flow observed in individual capillaries in layers 2 through 4 of rat neocortex," Proc. Natl. Acad. Sci. 95, 15741-15746 (1998).

15. V. J. Srinivasan et al., "Rapid volumetric angiography of cortical microvasculature with optical coherence tomography," Opt. Lett. 35, 43-45 (2010).

16. A. G. Hudetz, "Blood flow in the cerebral capillary network: a review emphasizing observations with intravital microscopy," Microcirc. N. Y. N. 4, 233-252 (1997).

17. A. G. Hudetz et al., "Effects of hypoxia and hypercapnia on capillary flow velocity in the rat cerebral cortex," Microvasc. Res. 54, 35-42 (1997).

18. A. G. Hudetz, G. Fehér, and J. P. Kampine, "Heterogeneous autoregulation of cerebrocortical capillary flow: evidence for functional thoroughfare channels?" Microvasc. Res. 51, 131-136 (1996).

19. A. G. Hudetz et al., "Video microscopy of cerebrocortical capillary flow: response to hypotension and intracranial hypertension," Am. J. Physiol. 268, H2202-H2210 (1995).

20. A. G. Hudetz et al., "Effect of hemodilution on RBC velocity, supply rate, and hematocrit in the cerebral capillary network," J. Appl. Physiol. 87, 505-509 (1999)

21. B. B. Biswal and A. G. Hudetz, "Synchronous oscillations in cerebrocortical capillary red blood cell velocity after nitric oxide synthase inhibition," Microvasc. Res. 52, 1-12 (1996).

22. J. Seylaz et al., "Dynamic in vivo measurement of erythrocyte velocity and flow in capillaries and of microvessel diameter in the rat brain by confocal laser microscopy," J. Cereb. Blood Flow Metab. Off. J. Int. Soc. Cereb. Blood Flow Metab. 19, 863-870 (1999).

23. E. Pinard et al., "Penumbral microcirculatory changes associated with peri-infarct depolarizations in the rat," Stroke J. Cereb. Circ. 33, 606-612 (2002).

24. M. L. Schulte, J. D. Wood, and A. G. Hudetz, "Cortical electrical stimulation alters erythrocyte perfusion pattern in the cerebral capillary network of the rat," Brain Res. 963, 81-92 (2003).

25. E. B. Hutchinson et al., "Spatial flow-volume dissociation of the cerebral microcirculatory response to mild hypercapnia," Neuroimage 32, 520-530 (2006).

26. B. Stefanovic et al., "Functional reactivity of cerebral capillaries," J. Cereb. Blood Flow Metab. 28, 961-972 (2007).

27. M. Unekawa et al., "Frequency distribution function of red blood cell velocities in single capillaries of the rat cerebral cortex using intravital laser-scanning confocal microscopy with high-speed camera," Asian Biomed. Res. Rev. News 2, 203-218 (2008).

Biographies for the authors are not available. 